\title{
DIGITALLY GLOBALIZED LANDSCAPES. THE EVOLUTION OF DATAVEILLANCE PRACTICES: DIGITAL DISTRIBUTION AND STREAMING PLATFORMS
}

\begin{abstract}
The study is an analysis of data gathering and data processing practices pertaining major PC digital distribution and streaming platforms, that is Valve's Steam and Amazon's Twitch.tv. Selected case studies from different than Western perspectives on gaming culture and the use of technology were introduced to assess the globalized state of the industry. The study consists of three parts: first, it deals with the notion of digital spatiality. Second, it scrutinizes the persuasiveness of the multi-layer visual interfaces. Third, it moves into spaces beyond gameplay and addresses the double nature of modern dataveillance techniques which mediate between the visible and non-visible elements of the analyzed gaming service platforms. The methodological scaffolding of the work is based on digital surveillance studies and software studies approaches rooted in the visual aspect of the GUIs (graphical user interfaces).
\end{abstract}

Keywords: dataveillance, video games, gaming service platforms, Steam, Twitch.tv, space

\section{Overview}

This study is aimed at analyzing the evolution of data gathering and data processing practices pertaining major PC digital distribution and streaming platforms, that is Valve's Steam and Amazon's Twitch.tv. The goal of the text is to map the connections between the data-gathering and data-processing tools and the visual rhetoric of the interface. Considering the two major digital distribution platforms in the PC segment of the video games market will allow for a comparative analysis of the evolution of the visual media rhetoric, which, as I would argue, is gradually shifting towards a data-centered model. In other words, the study will investigate the relation between the nonvisibility (or at least obstructed visibility) of the data-gathering algorithms and the (partial and circumvented) visibility of the graphic interface. The proposed analysis consists of two parts.

First, I will look into the concepts of digital spatiality and dataveillance in relation to post-digitality and computational spatial practices. Second, I would deal with the 
question of how the visual architecture of the digital platforms' interface shapes the discourse of and about the games. Third, I would move into the assessment of spatial practices which traverse beyond the "flat" visual interface and provoke video games' fans to explore the space beyond the computer screen. In this category, the most crucial technologies are associated with live video streaming, which, in connection with mobile technologies, is nowadays the fastest growing branch of game-based digital entertainment. ${ }^{1}$

The following research is based predominantly on two platforms: Valve's digital distribution platform Steam and Amazon's live streaming video platform Twitch.tv. Both are among the most popular mediums in their respectable categories, with global market penetration and significant cultural impact which especially in case of Twitch.tv is not limited only to video games audiences. ${ }^{2}$ Selected case studies from different than Western perspectives on gaming culture and the use of technology were introduced to assess the globalized state of the industry.

\section{Digital spatiality and dataveillance in digital platforms}

The idea of video games as forms of spatial practice is not a new one, and it has been explored both from the perspective of a body space ${ }^{3}$, and the perspective of inhabited urban environment. ${ }^{4}$ Moreover, several game studies analyses engage with the spatial theories of Henri Lefebvre ${ }^{5}$, although the bulk of the research comments on the in-game digital spatiality, whereas the following research focuses also on the mediatization of non-digital gaming spaces. The embodied space has been analyzed through the idea of embodied corporeality ${ }^{6}$ which emerges through the mediated image of a fragmented body during live broadcasts on digital streaming platforms. The important notion here is that the spatial presence of human player's body is meant to

A. Kerr, Global Games: Production, Circulation and Policy in the Networked Era, New York: Routledge, 2017.

2 See T.L. Taylor, Watch Me Play: Twitch and the Rise of Game Live Streaming, Princeton, NJ: Princeton University Press, 2018.

3 B. Flynn, "Games as Inhabited Spaces", Media International Australia Incorporating Culture and Policy 2004, vol. 110, no. 1, pp. 52-61.

4 R. Álvarez, F. Duarte, "Spatial Design and Placemaking: Learning From Video Games", Space and Culture 2018, vol. 21, no. 3.

5 Ch. Chesher, "Navigating Sociotechnical Spaces: Comparing Computer Games and Sat Navs as Digital Spatial Media", Convergence: The International Journal of Research into New Media Technologies 2012, vol. 18, no. 3, pp. 315-330; M. Bonner, "On Striated Wilderness and Prospect Pacing: Rural Open World Games as Liminal Spaces of the Man-Nature Dichotomy", in: Proceedings of DiGRA 2018, 2018, http://www.digra.org/wp-content/uploads/digital-library/DIGRA_2018_paper_18.pdf; J. Janik, "(Re)Weave the Gameplay: Analysis of the Spatial Textures in Yandere Simulator", 2017.

6 S.L. Anderson, "Watching People Is Not a Game: Interactive Online Corporeality, Twitch.Tv and Videogame Streams", Game Studies 2017, vol. 17, no. 1, http:/gamestudies.org/1701/articles/anderson. 
be seen through the specific features of the platform's interface, thus "grounding videogames in the corporeal, embodied context". ${ }^{7}$ While the practices associated with videogames become increasingly depended on specific use of technology, and - to larger extent - digital and physical platforms, these technological amenities simultaneously act upon the elements of both digital and physical (embodied) imprint of their users. Gameplay analytics tools scrutinize and measure not only the in-game actions, but gather information on human behavioral patterns, personal preferences and real-life associations. Steam, one of the currently leading digital distribution platforms in the PC market, actively collects data on its customers' habits. Game metric tools such as SteamSpy enable everyone who has access to the Internet to check the most played titles on the most popular platforms, albeit the data gathering process is not limited to commercial purposes. David Berry in Postdigital Aesthetics states that "computational technologies direct us towards a passive trust in widely delegated, yet obfuscated, actions". ${ }^{8}$ The issue of players' agency in game studies has also historically been placed in the realm of aesthetics, with a Janet Murray's seminal book Hamlet on the Holodeck (updated in the 2016's edition) defining it as a "satisfying power to take meaningful action and see the results of our decisions and choices", through which a computer-mediated "world [...] is dynamically altered by our participation". ${ }^{10}$ In this classic take, agency differs from activity because of its connection to intentionality ${ }^{11}$ - if the outcome meets the users' individual design and is not merely forced by the imposed game rules, the true agency emerges. However, the meaning of agency can go beyond its understanding as a choice made freely within the specific rule system. As is argued by Karen and Joshua Tanenbaum, agency is tied to a particular "commitment to meaning", that is the willingness to pursue the "expressive intent, and receiving a satisfying response to that intent". ${ }^{12}$ The interesting part of this theory is that agency can be expressed through cognitive process or players' actions. Interacting with the interfaces of digital distribution and streaming platforms are nowadays becoming an increasingly integral part of gaming experience, and the elements encouraging the users' agency are aimed predominantly towards the simulation of intention rather than committing to a concrete action. For example, indie developers regularly encourage those who wish to support their new game to include the upcoming title in their "wishlist"- this act is expressing an intent (thus informing the data-gathering algorithm about a potential customer), but it

Ibid.

8 D.M. Berry, M. Dieter, eds., Postdigital Aesthetics: Art, Computation And Design, Basingstoke: Palgrave Macmillan, 2015, p. 5.

9 J. Murray, Hamlet on the Holodeck: The Future of Narrative in Cyberspace, New York \& London: The Free Press, 2016, p. 123.

$10 \quad$ Ibid., p. 124.

11 Ibid., p. 125.

12 K. Tanenbaum, J. Tanenbaum, "Commitment to Meaning: A Reframing of Agency in Games", in: Digital Arts and Culture Conference, Irvine, 2009. 
is a non-binding action, as it does not oblige the player to purchase the game once it launches on Steam. Similar mechanisms of engagement can be traced on Twitch.tv or YouTube platforms, where the number of "followers" does not translate into any form of spectators' commitment, and even paid subscriptions (in the case of streaming platforms) can be transferred from one user to another based just on their presence in the chat, without any form of consent from the "gifted" recipients. It can be argued the digital platforms serving computer entertainment require passivity, but not without agency. Quite the contrary, they crave users' agency, albeit with the option to control it and profit from the dissemination of fans' labor through various channels. Thus, one of the default modes of engagement with the digital platforms is to exert the various forms of non-binding, purely intentional agency-users are merely signaling the potentiality to act instead of committing, or in some instances - agreeing to it. There are many similarities in the interface design and mechanisms of algorithm-based recommendation systems between game-centered streaming platforms and services like YouTube ${ }^{13}$, although Twitch.tv offers more options for cross-software integration, including the databases accessed via user accounts from different services (such as Activision Blizzard's Battle.net). Given the community-building aspect of streaming platforms ${ }^{14}$, such options become a powerful gateway for the implementation of various dataveillance practices. The seminal argument made by Julian Kücklich in $2005^{15}$ was about modders being a non-paid driving force behind the video games entertainment; nowadays the issue seems to switch from monetary to ownership-related. When the Steam workshop, a service within the Valve's digital distribution platform, planned to launch the paid version of mods for The Elder Scrolls V: Skyrim, the community's concerned reaction was about which entities have the right to distribute, preserve and govern the mods, not so much about whether the work associated with them deserves to be compensated in monetary form. ${ }^{16}$ This example tells the story about the proliferation of the space. The economy of space has many aspects: server space determines which data are to be circulated, and which are doomed to vanish; hard drives space, albeit relatively cheap, calls the gamers to make a choice each time they allocate its resources to download and play a game. The latter issue is magnified through the increasing indispensability of SSDs (solid state drives), oftentimes providing a crucial asset in the performance of the online games. The key resources are the server-client access time and users' time, measured not only in hours spent playing a given title, but also in time devoted to consuming

13 T. Wulf, F.M. Schneider, S. Beckert, "Watching Players: An Exploration of Media Enjoyment on Twitch", Games and Culture 2018, pp. 1-19, https://doi.org/10.1177/1555412018788161.

14 T.L. Taylor, op. cit.

15 J. Kücklich, "Precarious Playbour: Modders and the Digital Games Industry", The Fibreculture Journal 2005, no. 5, http://five.fibreculturejournal.org/fcj-025-precarious-playbour-modders-and-the-digital-games-industry/.

16 A. Paras, "Gabe Newell Talks about New Modding Policy: 'Our Goal Is to Make Modding Better"', Wccftech 2015, https://wccftech.com/gabe-newell-talks-modding-policy-goal-modding/. 
its paratexts: online streams, YouTube videos or even community-shared memes. The impact of streaming services and digital platforms on the video game market is steadily increasing ${ }^{17}$, and the key aspect of it is connected to the willingness of users to provide various forms of input: from personal data to creative digital work.

Dataveillance practices, that is methods of surveillance executed with the use of data gathering and processing procedures, are commonly used in the video games market. What is more, the knowledge of being tracked arguably became incorporated in the gameplay mechanics, especially in the form of in-game achievements system. ${ }^{18}$ The spatial dimension of dataveillance can manifest through coercing players into community-oriented activity. The 2016's iteration of the famous Doom computer game franchise featured separate set of achievements for successfully designing and sharing personally crafted maps with the built-in SnapMap system. Creative construction of the in-game space goes hand in hand with the subjugation of this space to the logic of marketing: when the player's activity is confirmed by the algorithm, the achievement becomes visible to the users' "friends" on Steam platform, thus advertising this feature to other potential customers. Most notably, there is no option to opt-out from earning and displaying achievements once one is registered on a given digital platform, and these systems encompass both PCs and console segments of the market.

Alex Cybulski argues that in understanding videogame surveillance, it is crucial to conceptualize them as simulations, where activities are "represented through the execution of code". ${ }^{19}$ The close interconnection between human actions and algorithm processing does not, however, mean the direct translation. For example, dataveillance algorithms governing achievement systems may fall to provoke proper responses from the level of graphic user interface, and specific in-game triggers are also susceptible to errors, especially given the often complicated, hierarchical structure of conditions set for activating a given response. The ways in which code operates during the gameplay usually remains obfuscated to the end-users, even though the hidden labor of data accumulation and processing sometimes wants to be seen and manifests in the GUI (graphical user interface) of digital distribution and streaming platforms. The in-game Steam overlay features the tools to make and archive screenshots, connect people via the group and matchmaking invitations, and in general provides an additional graphical and functional amenity for the users. However, there is also one crucial feature enabled through Steam overlay - the ability to complete in-

17 M.R. Johnson, J. Woodcock, "The Impacts of Live Streaming and Twitch.Tv on the Video Game Industry", Media, Culture and Society 2018, pp. 10-28, https://doi.org/10.1177/0163443718818363; K. Pires, G. Simon, "YouTube Live and Twitch: A Tour of User-Generated Live Streaming Systems", in: Proceedings of the $6^{\text {th }}$ ACM Multimedia Systems Conference, New York: ACM, 2015, pp. 225-230.

18 See C. O'Donnell, "Getting Played: Gamification, Bullshit, and the Rise of Algorithmic Surveillance", Surveillance and Society 2014, vol. 12, no. 3, pp. 349-359.

19 A.D. Cybulski, "Enclosures at Play: Surveillance in the Code and Culture of Videogames", Surveillance and Society 2014, vol. 12, no. 3, pp. 427-432. 
game transactions involving real money stored on the Steam account. The connection between economy, computer-related entertainment and automated data accumulation transcends the traditional borders of digitality and permeates other areas of everyday life. Steam overlay appears as something that is seemingly unobtrusive, works "behind" the series of other visual interfaces, and remains necessary to transfer certain forms of agency from the modern-day banking to the economic dimension of a digital platform.

\section{Persuasive visual architecture and interfaces}

Commenting on Bruno Latour's notions of digitality, Berry observes that "digital is digital less as a domain and more as a set of practices - or, perhaps, that the digital is better understood as an inscription device for capturing, or remediating, practice" ${ }^{20}$. I would argue that the persuasive rhetoric of game spaces visuality is building on that exact premise - that the digital serves as a gateway to the realm of other practices, and the digitalized information forms a basis for subsequent remediation, reproduction or creative, paratextual interpretation. In other words, the current cultural landscape of gaming is defined not so much by the digitality of the separate medium artifacts (the games), as it is shaped by the politics of showing/obfuscating of the digitalized experiences which, after remediation through digital platforms and streaming services, become the focal points of gaming discourse. Thus, game developers who wish to achieve success must cope with the aforementioned platforms to establish a common ground, a specific media language to accommodate the needs for both digital platform advertisements and promotional campaigns and communication methods specific to the medium of streaming platforms. Similar conclusions can be drawn in relation to other forms of digital cultural production, as the "multisided platform markets" 21 are becoming the staple of day-to-day cultural content production and circulation. The application of Latour's actor-network theory to platform and video game studies brought a re-definition of the previously mentioned notions of agency, now understood as "dislocated production of differences", which inevitably manifests in action and introduces change..$^{22}$ This framework is congruent with the logic of platform: "for Latour attributes are not inert qualities, but performances" ${ }^{23}$, and non-human actors are inevitably active agents in the operations performed on digital platforms and services on everyday basis. However, the analysis of these

20 D.M. Berry, M. Dieter, eds., op. cit., p. 47.

${ }_{21}$ D.B. Nieborg, T. Poell, "The Platformization of Cultural Production: Theorizing the Contingent Cultural Commodity", New Media and Society 2018, vol. 20, no. 11, pp. 4275-4292, https://doi. org/10.1177/1461444818769694.

22 D. Muriel, G. Crawford, "Video Games and Agency in Contemporary Society", Games and Culture 2018, https://doi.org/10.1177/1555412017750448.

23 G. Harman, Prince of Networks: Bruno Latour and Metaphysics, Melbourne: re.press, 2009, p. 45. 
actions and their implication for each of the entities involved in the process would benefit from going beyond the traditional ANT toolkit and looking into the relation between the interface and digital as well as non-digital corporeality. The first step towards such analysis is the assessment of data visualization methods, and the second - an investigation of how they are intertwined with digitalized physical space.

Data visualization methods are used as a countermeasure to the review bombing and other crowd-based methods of influencing the discourse of and about the digital texts of culture. In response to this practice, Valve introduced a data visualization tool: diachronic histograms which allow for checking the positive vs. negative reviews ratio during a given period. The possible caveats of such solution are twofold. First, the average customer would be hard-pressed to delve deep enough into the Steam's interface to actually see the histogram. The main page of each game still displays the recommended/not recommended ratio of users' votes, without the information that the histogram provides. Second, it is a method of data visualization, and as such, according to Alexander Galloway, it does not necessarily bring any information, as "any visualization of data must invent an artificial set of translation rules that convert abstract number to semiotic sign". ${ }^{24}$ Translating the dataset-in this case the number of negative and positive reviews in a given time frame-into a visual format of an easily readable graph, arguably cannot provide the users with information necessary to interpret it. With binary yes/no mechanism of labeling and counting users' feedback, the content of each comment is lost in the process of data-to-visual transition. What is more, the modern distribution of video games relies heavily on the post-production element of games' life cycle, and histograms are oblivious to the actual reasons behind the fluctuations of votes. Controversial patch content or unpopular developer's decision may heavily influence the histogram regardless of the - necessarily belated - interpretation of it being a case of a review bombing. Information extracted from automated dataveillance becomes actual information after filling the holes by a human agent, that is after the process of human interpretation completes its work, taking into consideration or omitting facts and other intertwined datasets according to the desired end goal.

With Chinese' Tencent being one of the largest game-related media conglomerates in $2016^{25}$, Western high-budget companies are increasingly more interested in implementing business and surveillance strategies inspired by the recent Asian developments in the field. The advent of Chinese facial recognition programs, with the notable example of Megvii's Face++, may seem like the decisive step in surveillance techniques after the world-wide introduction of biological passports. Many of the digital surveillance solutions which are introduced in relation to video games are based on experiences with mobile technology, as mobile gaming business is booming in the Asian region. Even triple-A developers like Activision Blizzard maintain

24 A.R. Galloway, The Interface Effect, Cambridge: Polity Press, 2012, p. 83.

25 A. Kerr, op. cit. 
their development decisions based on the dominating trends on Asian markets, with newest iteration of the popular Diablo franchise to be released in the mobile-only format, which sparked controversy among Western fans and contributed to the overall financial difficulties for the company. ${ }^{26}$ It is worth to note that the seemingly radical business decisions involving the expansion into alternative platforms and abandoning traditional PC- and console-centered models of games development are a direct reaction to the recent shifts in economic trends, and are not limited to Blizzard. The dataveillance interfaces in gaming technologies are a serious perspective for the years to come, and data recognition software has just entered the digital spatiality of Asian gamers. An example of this phenomenon comes from the aforementioned company Tencent, which introduced the age restriction on one of its most popular titles, Honour of Kings, allegedly in response to the growing gaming addiction problem of Chinese youth. From September 2017, the system has been linked to the database supervised by the China's Ministry of Public Security ${ }^{27}$, which effectively means that the face recognition algorithms will be used more frequently to verify the age of gamers. Successful passing of the face recognition test equals nearly unlimited gameplay time, whereas minors under the age of 18 are restricted to one hour of daily gameplay. It is important to note that these limitations were introduced in cooperation with Chinese government, which sets to implement more decisive control measures over the video games industry. The gamer is no longer devoid of physical features, and video games long ago ceased to be an escape from material reality of everyday life, if they ever offered such opportunity in the first place. The corporeal turn in game-related dataveillance goes hand-in-hand with corporeal turn in how games nowadays function as digital artifacts - how they are consumed and which elements of their interface are to be enacted with human bodies as meaningful contributors.

\section{Streaming platforms and space outside the gameplay}

Game-related live streaming video platforms are not devoid of material components directly related to the physical space in which the streamer performs her broadcast. Anderson ${ }^{28}$ speaks about the notion of "interactive online corporeality", referring to the set of technical affordances and Twitch.tv features which are showcasing the human body as crucial element of the streaming process. This type of

26 W. Colville, "Activision Blizzard Falls 10 Percent after Reporting Decline in Monthly Active Users", CNBC, 2018, https://www.cnbc.com/2018/11/08/activision-blizzard-shares-fall-10-percent-after-reporting-decline-in-monthly-active-users.html.

${ }_{27}$ K. Webb, "Video Game Addiction Has Sparked a Culture War in China-and It's Having Huge Repercussions for the World's Biggest Video Game Maker", Business Insider 2018, https://www. businessinsider.com/tencent-age-restrictions-identity-verification-china-video-games-2018-11? IR=T. S.L. Anderson, op. cit. 
corporeality emerges in both personal and impersonal live streams ${ }^{29}$, although it can be argued that corporate-sponsored coverage of live events mediates the non-digital space of e-sports arenas or other public venues in a different way than is possible in the personal streams. ${ }^{30}$ In addressing the issue of dataveillance phenomena pertaining streaming platforms and the mediation of the space which resides outside the area of in-game gameplay, I will assess both of the aforementioned types of online broadcasting.

Video games were one of the examples Lev Manovich used in his renown take on the language of new media. Among the key new media properties, he enlisted numerical representation modularity, additionally stating that "they should be considered not as some absolute laws but rather as general tendencies of a culture undergoing computerization". ${ }^{31}$ Video games not only can be represented in the numerical formwhich would be the obvious take from Manovich's analysis - but they also have the ability to record human activity through the various forms of digital imprints, which serves as a track record of human-computer interaction. Fulfilling the modularity principle effectively means that there are many (possibly interchanging) layers and elements of a video game, and each gameplay instance potentially leaves a series of traceable digitalized histories, highly susceptible to subsequent dataveillance practices. In other words, games' save files and gameplay recordings all share the ability to become unique artifacts, preserving data on players' behavior, intricacies, specific modes of interaction or used third-party programs or peripheries.

A few years after the publication of The Language of New Media Manovich wrote an article on augmented space, this time claiming that "connection between surveillance/monitoring and assistance/augmentation is one of the key characteristics of the high-tech society". ${ }^{32}$ In this type of society, physical space becomes a data space through the process of extraction or augmentation. Similarly, the properties pertinent to the physical space of gameplay can be retraced using the dataveillance techniques, in a process reverse to the one described by Manovich. The most notable examples

29 See V.-M. Karhulahti, "Prank, Troll, Gross and Gore: Performance Issues in Esport Live-Streaming", in: Proceedings of $1^{\text {st }}$ International Joint Conference of DiGRA and FDG, 2016, http://www.digra. org/wp-content/uploads/digital-library/paper_110.compressed.pdf. Karhulahti distinguishes between personal live-streaming ("the practice of live-streaming one's personal performance (game-related or not) via platforms like Twitch.tv") and impersonal live-streaming, which refers to "a parallel [to personal live-streaming] practice structured by a third-party organization over the individual performers and their local audience".

30 For further reference regarding impersonal live streaming in e-sports venues, see M. Felczak, "Narratives of Spectatorship: E-Sports in Poland", in: New Perspectives in Game Studies: Proceedings of the Central and Eastern European Game Studies Conference Brno 2014, 2014, pp. 109-123, http:// gamestudies.cz/wp-content/uploads/ceegs-online-final-1.pdf.

31 L. Manovich, The Language of New Media, Cambridge: MIT Press, 2001, p. 49.

32 L. Manovich, "The Poetics of Augmented Space", Visual Communication 2006, vol. 5, no. 2, pp. 219-240, p. 222. 
involve the documented speedruns ${ }^{33}$, especially these which achieved particularly impressive results. Previously, speedruns were recorded with the help of third-party software, and then scrutinized by the community to prove their validity. There are many categories and forms of speedruns, but for the sake of this research it is important to assess just one of their crucial features: they should be done (and recorded) in one gameplay session, and the practice of splitting the run into fragments is generally frowned upon and considered cheating. Nowadays, with the advent of Twitch.tv and similar streaming services, it is seemingly easy to prove such frauds, as the live performance in front of the public should ensure that the fair play rules are observed. However, the advances in recording technologies enable streamers to effortlessly display an overlay of a pre-recorded, "spliced" segment of the particularly troublesome portion of the speedrun, thus cheating both the viewers and - in some regard-also the GUI of the streaming platform. ${ }^{34}$ This issue is potentially interesting from several perspectives. First, it proves the opacity and conventionalization of the mediated spaces: the digital in-game space, the streaming platform's interface, and the physical gameplay space surrounding the streamer. Second, such attempts at cheating can be subsequently divulged by the same dataveillance techniques which initially enabled the emergence of speedrunning as a cultural practice. Frame-perfect gameplay, taking advantage of carefully researched glitches, using data mining tools to find software shortcuts in the game engines all contribute to the final performance of a human actor who plays the game as efficiently as possible in front of the internet audience. At present, streamer cameras are not uncommon, and popular events such as Awesome Games Done Quick ${ }^{35}$ feature several live camera views encompassing both physical space of the venue and digital space of the game. When a speedrun attempt is scrutinized, it is measured against possible human performance, not the optimal datamined strategies. In speedrunning, digital surveillance shows its double dimension: a protocol on how to recognize a human player is juxtaposed with data from numerous performances as well as technical limitations of each speedrunned title.

In his research on Steam and Twitch.tv network visualizations, Enrico Gandolfi defines his object of study as "gaming service platforms, that is, multi-function online

33 Speedrunning can be defined as "the practice of players or 'runners' attempting to 'travel' from a game's opening state at its first necessary button input to the game's conclusion at its last necessary button input in the smallest amount of time possible". See: R. Scully-Blaker, "A Practiced Practice: Speedrunning through Space with de Certeau and Virilio", Game Studies 2014, vol. 14, no. 1, http:// gamestudies.org/1401/articles/scullyblaker.

34 This was the case of Paper Mario: The Thousand-Year Door speedrun attempt by a player nicknamed Chibi who used the aforementioned action replay cheat in one of his live streams on Twitch.tv. For further details, see https://forums.mylzh.net/forum/lzuruha-game-forums/speed-running-consoles/ speed-running-forums/newer-consoles-discussion/170475-chibisrl-cheating-with-action-replay-inpaper-mario-ttyd.

35 A charity speedrun marathon event which gathers money supporting the Prevent Cancer Foundation. 
environments that allow users to experience digital games beyond the mere play", ${ }^{36}$ The component that lies beyond the sphere of play refers to various spaces and dimensions, both of digital and physical (albeit mediated through the platforms' interfaces) forms.

\section{Conclusion}

In April 2018, Valve Corporation decided to hide the information about users' owned games library in the Steam's Web Application Programming Interface (API) unless they explicitly enable such option. With the additional changes in the Steam store API, this rendered one of the key datamining services, SteamSpy, practically useless. However, the old algorithms and methods got replaced by others, and the service continues to be available to the public_-gamers and developers alike can easily track the games' popularity, number of owned copies and major trends pertaining e.g. the most used languages on that platform. Video games have become more diversified in their modes of consumption and leading distribution approaches. Dataveillance techniques permeate the industry on multiple levels, and their importance becomes evident in mediating and transferring the users' experience. Digital distribution platforms seek information about individual players' experiences, but some of the data is directly transferred into visual form of GUI feedback for commercial or persuasive purposes. Streaming services mediate the physical, audience-focused space through the layers of visual interfaces, introducing forms of interactivity which are alternative to the sole act of playing games. Both types rely on dataveillance methods constantly shifting between what is visible and not visible from the perspective of an average user. The current landscape of video games is shaped through the globalization and proliferation of its platforms, in terms of hardware and software alike. Despite the digitalization of video games industry, the notions of spatial division become increasingly important in determining the access to certain titles, with new proprietary platforms (such as Epic Games Store) pushing for deals to secure exclusive rights to distribute the most sough-after products. The power to control the digital ownership comes with the much higher power of computation, which enables the implementation of data aggregating algorithms and monitoring players' behaviors.

\section{Bibliography}

Álvarez R., Duarte F., “Spatial Design and Placemaking: Learning from Video Games”, Space and Culture 2018, vol. 21, no. 3.

36 E. Gandolfi, "Enjoying Death among Gamers, Viewers, and Users: A Network Visualization of Dark Souls 3's Trends on Twitch.Tv and Steam Platforms", Information Visualization 2017, vol. 17, no. 3 , p. 219 , https://doi.org/10.1177/1473871617717075. 
Anderson S.L., "Watching People Is Not a Game: Interactive Online Corporeality, Twitch.Tv and Videogame Streams", Game Studies 2017, vol. 17, no. 1, http://gamestudies.org/1701/ articles/anderson.

Berry D.M., Dieter M., eds., Postdigital Aesthetics: Art, Computation and Design, Basingstoke: Palgrave Macmillan, 2015.

Bonner M., "On Striated Wilderness and Prospect Pacing: Rural Open World Games as Liminal Spaces of the Man-Nature Dichotomy", in: Proceedings of DiGRA 2018, 2018, http:// www.digra.org/wp-content/uploads/digital-library/DIGRA_2018_paper_18.pdf.

Chesher Ch., "Navigating Sociotechnical Spaces: Comparing Computer Games and Sat Navs as Digital Spatial Media", Convergence: The International Journal of Research into New Media Technologies 2012, vol. 18, no. 3, pp. 315-330.

Colville W., “Activision Blizzard Falls 10 Percent after Reporting Decline in Monthly Active Users", CNBC, 2018, https:/www.cnbc.com/2018/11/08/activision-blizzard-shares-fall-10-percent-after-reporting-decline-in-monthly-active-users.html.

Cybulski A.D., "Enclosures at Play: Surveillance in the Code and Culture of Videogames", Surveillance and Society 2014, vol. 12, no. 3, pp. 427-432.

Felczak M., "Narratives of Spectatorship: E-Sports in Poland", in: New Perspectives in Game Studies: Proceedings of the Central and Eastern European Game Studies Conference Brno 2014, 2014, pp. 109-123, http://gamestudies.cz/wp-content/uploads/ceegs-online-final-1. pdf.

Flynn B., "Games as Inhabited Spaces”, Media International Australia Incorporating Culture and Policy 2004, vol. 110, no. 1, pp. 52-61.

Galloway A.R., The Interface Effect, Cambridge: Polity Press, 2012.

Gandolfi E., "Enjoying Death among Gamers, Viewers, and Users: A Network Visualization of Dark Souls 3's Trends on Twitch.Tv and Steam Platforms", Information Visualization 2017, vol. 17, no. 3, pp. 218-238, https://doi.org/10.1177/1473871617717075.

Harman G., Prince of Networks: Bruno Latour and Metaphysics, Melbourne: re.press, 2009.

Janik J., "(Re)Weave the Gameplay: Analysis of the Spatial Textures in Yandere Simulator", 2017.

Johnson M.R., Woodcock J., "The Impacts of Live Streaming and Twitch.Tv on the Video Game Industry", Media, Culture and Society 2018, pp. 10-28, https://doi.org/0.1177/ 0163443718818363.

Karhulahti V.-M., "Prank, Troll, Gross and Gore: Performance Issues in Esport Live-Streaming”, in: Proceedings of $1^{\text {st }}$ International Joint Conference of DiGRA and FDG, 2016, http://www.digra.org/wp-content/uploads/digital-library/paper_110.compressed.pdf.

Kerr A., Global Games: Production, Circulation and Policy in the Networked Era, New York: Routledge, 2017.

Kücklich J., "Precarious Playbour: Modders and the Digital Games Industry", The Fibreculture Journal 2005, no. 5, http://five.fibreculturejournal.org/fcj-025-precarious-playbour-modders-and-the-digital-games-industry/.

Manovich L., The Language of New Media, Cambridge: MIT Press, 2001.

Manovich L., "The Poetics of Augmented Space", Visual Communication 2006, vol. 5, no. 2, pp. 219-240.

Muriel D., Crawford G., "Video Games and Agency in Contemporary Society", Games and Culture 2018, https://doi.org/10.1177/1555412017750448. 
Murray J., Hamlet on the Holodeck: The Future of Narrative in Cyberspace, New York \& London: The Free Press, 2016.

Nieborg D.B., Poell T., "The Platformization of Cultural Production: Theorizing the Contingent Cultural Commodity", New Media and Society 2018, vol. 20, no. 11, pp. 4275-4292, https://doi.org/10.1177/1461444818769694.

O’Donnell C., "Getting Played: Gamification, Bullshit, and the Rise of Algorithmic Surveillance", Surveillance and Society 2014, vol. 12, no. 3, pp. 349-359.

Paras A., "Gabe Newell Talks about New Modding Policy: 'Our Goal Is to Make Modding Better'”, Wccftech 2015, https://wccftech.com/gabe-newell-talks-modding-policy-goal-modding/.

Pires K., Simon G., "YouTube Live and Twitch: A Tour of User-Generated Live Streaming Systems", in: Proceedings of the 6th ACM Multimedia Systems Conference, New York: ACM, 2015, pp. 225-230.

Scully-Blaker R., "A Practiced Practice: Speedrunning through Space with de Certeau and Virilio", Game Studies 2014, vol. 14, no. 1, http://gamestudies.org/1401/articles/scullyblaker.

Tanenbaum K., Tanenbaum J., "Commitment to Meaning: A Reframing of Agency in Games", in: Digital Arts and Culture Conference, Irvine, 2009.

Taylor T.L., Watch Me Play: Twitch and the Rise of Game Live Streaming, Princeton, NJ: Princeton University Press, 2018.

Webb K., "Video Game Addiction Has Sparked a Culture War in China-and It's Having Huge Repercussions for the World's Biggest Video Game Maker", Business Insider 2018, https://www.businessinsider.com/tencent-age-restrictions-identity-verification-china-video-games-2018-11? IR=T.

WulfT., Schneider F.M., Beckert S., "Watching Players: An Exploration of Media Enjoyment on Twitch”, Games and Culture 2018, pp. 1-19, https://doi.org/10.1177/1555412018788161. 